\title{
A produção científica brasileira no feminino*
}

\author{
Hildete Pereira de Melo** \\ André Barbosa Oliveira***
}

\begin{abstract}
Resumo
O crescimento da produção científica tem estimulado o surgimento de estudos para avaliar os processos de produção, difusão e uso dos conhecimentos científicos. Infelizmente, essa literatura não tem sido sensível à temática de gênero, ignorando que mulheres $e$ homens têm trajetórias diferenciadas e que desvelar as mulheres no mundo científico e tecnológico exige um esforço específico. A hipótese da neutralidade científica, de fato, iguala os que não são iguais no acesso às carreiras científicas $e$ tecnológicas. Este artigo tem como objetivo preencher esta lacuna $e$ analisar através da utilização do indicador "medida de atividade", a intensidade da atividade científica das mulheres e homens no País; bem como, através do indicador "medidas de colaboração", a cooperação existente na produção científica nacional sob o prisma de gênero.
\end{abstract}

Palavras-chave: Gênero, Indicadores, Produção Científica.

\footnotetext{
* Recebido para publicação em março de 2006, aceito em maio de 2006.

*** Professora da Faculdade de Economia da Universidade Federal Fluminense, Niterói, Rio de Janeiro. hildete@economia.uff.br

**** Graduando de Economia, bolsista de iniciação científica, Universidade Federal Fluminense, Niterói, Rio de Janeiro. andrebosn@hotmail.com
} 
A produção científica brasileira no feminino

Brazilian Scientific Production in the Feminine

\begin{abstract}
The growth of scientific production has stimulated studies which analyse the processes of production, diffusion and use of scientific knowledge. Unfortunately, this literature has not been alert to gender issues; it has neglected the fact that men and women have different trajectories, and that to unveil women in the scientific and technological world demands a specific effort. The hypothesis of scientific neutrality effectively equates the unequals regarding access to scientific and technological careers. The present study aims to fill this gap and to analyse, through an activity indicator, the intensity of scientific activity of men and women in Brazil; and, through a collaboration indicator, the existing co-operation between genders in national scientific production.
\end{abstract}

Key Words: Gender, Indicators, Scientific Production. 
Hildete Melo e André Oliveira

\section{Apresentação do tema}

Um indicador importante da qualidade do trabalho dos cientistas é a capacidade dos pesquisadores de propor soluções para os problemas científicos e tecnológicos ou interpretar a realidade sócio-cultural. Para avaliar essa questão, governos $e$ pesquisadores utilizam a produção científica de cada país para conhecer o desempenho dos integrantes dos sistemas científicos e tecnológicos. Seja para conhecer as pesquisas sobre a natureza $e$ os determinantes dos processos de produção - difusão e uso de conhecimentos científicos, tecnologia e inovação -, seja para formular e avaliar políticas públicas.

Um dos trabalhos pioneiros dessa abordagem foi o artigo de Coles e Eales (1917), que estudaram a história da anatomia através de uma análise estatística da literatura científica dessa área do conhecimento e, segundo Leta e Cruz (2003), inauguraram esse tipo de análise - a bibliometria. ${ }^{1}$ Lentamente esse tipo de abordagem foi consolidando-se e nos anos 1970 surgiram revistas especializadas no assunto, tais como Scientometrics, Research Policy, Research Evaluation, Science and Public Policy, que passaram a divulgar estudos quantitativos da literatura baseados nesses indicadores. $\mathrm{Na}$ atualidade há uma grande demanda dos periódicos brasileiros na busca de indexação em bases de dados internacionais, bem como nas nacionais. A indexação significa controle da excelência do trabalho e aumenta o prestígio dos próprios periódicos e dos(as) autores(as), na medida em que cada base de dados define critérios rígidos para o processo de avaliação a que são submetidos os periódicos e estes são constantemente reavaliados. Algumas bases são públicas e de acesso livre, outras privadas e as informações dispendiosas.

No Brasil, nas duas últimas décadas, a produção científica cresceu extraordinariamente: passou de 2.609 publicações

\footnotetext{
${ }^{1}$ Leta e Cruz (2003) definem esse tipo de análise como "bibliometria", termo criado por Pritchard em 1969 como o uso de métodos matemáticos e estatísticos aplicados à análise de livros, artigos e outros meios de comunicação.
} 
A produção científica brasileira no feminino

em 1981, em todas as áreas do conhecimento, para 12.015 publicações em 1999 (FAPESP, 2002). ${ }^{2}$ Esta produção é extremamente concentrada na região Sudeste do Brasil, onde se localiza a maior parte das instituições universitárias, os programas de pós-graduação $e$ os pesquisadores nacionais, como demonstram os dados dessa pesquisa. ${ }^{3}$

O crescimento da produção científica tem estimulado o surgimento de estudos a respeito do assunto, seja criando indicadores de avaliação, com o objetivo de monitorar os processos de produção, difusão e uso dos conhecimentos científicos e tecnológicos, seja realizando comparações internacionais para melhor avaliar a produção nacional (Viotti \& Macedo, 2003). Infelizmente, essa literatura não tem sido sensível à temática de gênero. Os excepcionais estudos sobre a produção científica brasileira e paulista editados pela FAPESP (2001), UNICAMP (2003) e os Indicadores Nacionais de Ciência \& Tecnologia - 2002 do Ministério de Ciência e Tecnologia (MCT, $2004)^{4}$ não contemplaram esse recorte. Esses estudos ignoraram que mulheres e homens têm trajetórias diferenciadas e que é necessário conhecer os dados por sexo das situações analisadas para compreender o papel de cada um no mundo científico e tecnológico. Tal critério, sob aparente neutralidade, de fato, iguala os que não são iguais no acesso às carreiras científicas $e$ tecnológicas.

Este artigo, ainda de forma embrionária, tem como objetivo preencher esta lacuna $e$ analisar, através da utilização do indicador "medida de atividade", o número de artigos científicos publicados como indicador da intensidade da atividade científica no país; bem como, através do indicador medidas de colaboração, a

2 A base de dados do Institute for Scientific Information (ISI) também registra esse aumento: em 1981 as publicações nacionais eram 0,28\% do total da literatura mundial e em 1999 representam 1,02\%. (apud Fapesp, 2001].

3 Sobre o assunto, ver De Meis, L., \& Leta, J., 1996 e Guimarães, R., 2002.

4 Ver Viotti e Macedo, 2003. 
cooperação existente na produção científica nacional sob o prisma de gênero (Viotti, 2003). Na última década cresceu o número de mulheres realizando atividades científicas e há uma nítida tendência do avanço dessas pesquisadoras na direção da maior qualificação $e$ habilitadas a participar soberanamente, e não de forma subordinada, nos grupos de pesquisa nacionais. Urge mensurar a produção científica por sexo no Brasil para aumentar a maior visibilidade feminina no sistema científico. Esta análise utilizou a base de dados brasileira Scientific Electronic Library Online (SciELO).

No campo multidisciplinar - feminismo e ciência nos últimos trinta anos - surgiu uma ampla literatura feminista sobre a ciência, seja questionando a pouca atenção dada nos estudos dos sistemas científicos e tecnológicos ao tema de gênero, seja pelo discurso do conhecimento científico nas suas diversas vertentes. ${ }^{5}$ Desde os anos 1970, com a segunda onda feminista do século XX, esta temática também ressoou no Brasil, no início, com as pesquisadoras da Fundação Carlos Chagas, espalhando-se, posteriormente, no meio acadêmico com a consolidação de vários núcleos de estudos de gênero. Particularmente o tema gênero e ciência ganham relevância nos anos 1990, com destaque para estudos dos grupos da Unicamp, Fiocruz, NEIM/UFBA e tantas outras pesquisadoras individuais que analisam a ausência das mulheres da História da Ciência no Brasil. ${ }^{6}$

\section{Metodologia do Estudo}

Este trabalho limita-se a analisar a produção científica nacional a partir da base de dados SciELO, desenvolvida pela Fundação de Amparo à Pesquisa do Estado de São Paulo (FAPESP) e o Centro Latino-Americano e do Caribe de Informação

5 Ver Harding, 1998; Schiebinger, 2001; Pérez Sedeno, 2001; Tabak, 2002; Costa e Sardenberg, 2002; Rodriguez-Sala e Zubieta, 2005.

6 Ver Lopes, 1992 e 1998; Velho e Leon, 1998; Tosi, 1998; Sardenberg, 2002; Leta, 2003; Melo e Casemiro, 2003. 
A produção científica brasileira no feminino

em Ciências da Saúde (BIREME), disponível a partir de 1997. ${ }^{7}$ O SciELO permite o acesso gratuito ao texto integral de um número restrito de periódicos nacionais, que na sua maioria não estão incluídos na Institute for Scientific Information (ISI), uma das mais importantes bases de dados internacional sobre o tema. Apesar de sua importância há lacunas na base do ISI para analisar a produção científica nacional, devido ao peso de determinadas publicações e a ausência de outras áreas do conhecimento, com uma maior participação das chamadas "ciências duras". A base de dados ISI privilegia a indexação de periódicos em língua inglesa em detrimento de revistas publicadas em países que não tem o idioma inglês como língua oficial. Também não admite a publicação de estudos regionais, o que penaliza o Brasil, pois o recorte regional é importante na produção local e representa um grande número de estudos e pesquisas realizados no território nacional.

Para suprir essas restrições, o SciELO foi organizado com o objetivo de promover a inclusão da produção literária científica brasileira no circuito internacional rumo a publicação eletrônica; é uma livraria eletrônica que agrupa um grande número de periódicos brasileiros, coleção de títulos individuais, que iniciou sua implantação em 1997 e atualmente é a melhor referência sobre o assunto disponível na literatura nacional. Nesta base de dados admite-se que o periódico seja indexado em uma ou mais áreas do conhecimento. Para evitar sobreposição de periódicos essa pesquisa usou a classificação de áreas do conhecimento do próprio SciElO: Ciências Agrárias, Ciências Biológicas, Ciências da Saúde, Ciências Exatas e da Terra, Ciências Humanas, Ciências Sociais Aplicadas, Engenharias e Lingüística, Letras e Artes.

A pesquisa mostrou que 23 periódicos estão classificados em mais de uma área do conhecimento. Para eliminar a dupla

7 Sobre o SciELO, ver a edição especial da Revista Ciência da Informação, 1998, vol.27, $\mathrm{n}^{\circ} 2$. 
contagem dos periódicos, ao fazer a análise quantitativa da produção científica pelas áreas do conhecimento foi feita uma escolha arbitrária entre as áreas do conhecimento a que os periódicos se enquadravam. Para ilustrar o procedimento, no caso dos Anais da Academia Brasileira de Ciências, que estava enquadrado em Ciências Biológicas, Ciências da Saúde, Ciências Agrárias, Ciências Exatas e da Terra, Ciências Humanas e Engenharias, neste trabalho, a revista foi classificada na área de Ciências Exatas e da Terra. A mesma dupla contagem ocorreu com o periódico Cadernos Pagu que estava classificado em Ciências Humanas, Sociais Aplicadas e da Saúde, arbitrou-se contabilizar esta publicação na área Ciências Humanas. O mesmo procedimento foi realizado para os outros 21 periódicos classificados em mais de uma área do conhecimento, de forma que cada periódico e seus artigos ficassem classificados em uma única área do conhecimento. Em janeiro de 2006, o SciELO catalogava 56.694 artigos publicados em 147 periódicos nacionais de diversas áreas do conhecimento no período de 1997/2005. ${ }^{8}$

A metodologia para avaliar a produção científica feminina indexada na biblioteca eletrônica SciELO foi realizada através de consulta ao site http://www.scielo.br ao longo dos meses de outubro de 2005 a janeiro de 2006. A partir da lista de periódicos por área de conhecimento foi selecionado o link para a página principal da revista. Dessa para página de acesso aos fascículos disponíveis do período e para a página contendo o sumário de cada fascículo e dessa obteve-se o acesso aos artigos, com seus autores e data de publicação. $\mathrm{O}$ trabalho de organização da base de dados deste artigo foi penoso devido à inexistência de discriminação por sexo na biblioteca SciELO, levando a classificar cada artigo em separado.

Organizadas as referências bibliográficas de cada área foi feito o seguinte procedimento: primeiro uma triagem dos prenomes femininos e masculinos, reconhecidos como tais pela

8 www.scielo.br, acessos 9-13 de janeiro de 2006. 
A produção científica brasileira no feminino

língua portuguesa. Como há uma grande coincidência dos prenomes utilizados pelos diversos autores, usou-se o programa Visual Basic para ordenar a classificação dos prenomes por sexo, segundo o uso comum na língua portuguesa. Atribuiu-se aos nomes masculinos o digito 1 e aos femininos o digito 0 , e a busca foi realizada para um número muito inferior do que estavam efetivamente listados. A cada rodada de identificação de um prenome, o programa atribuía o número correspondente ao sexo masculino ou feminino, e o "digito 2" para os indefinidos. Para estes últimos e para os prenomes diferentes ou estrangeiros foi feito um reconhecimento individual para cada caso. Para o conjunto dos prenomes, que podem ser usados por ambos os sexos ou abreviados, foi utilizado a Plataforma LATTES do CNPq para definir o sexo do autor(a). Além de demorado, esse método apresenta limitações, mas possibilitou resolver muitos casos duvidosos. A busca foi feita na base de dados LATTES em consultas individualizadas disponibilizadas pelo $\mathrm{CNPq}$ no site http://buscatextual.cnpq.br/ buscatextual/index.jsp.

A importância da Plataforma LATTES no sistema científico e tecnológico brasileiro é inegável. Há cerca de 77.649 pesquisadores cadastrados e 604.395 currículos enviados até 18 de outubro de 2005, felizmente a Plataforma LATTES discrimina o sexo nessa base de dados. A busca foi feita usando-se o nome e as iniciais do prenome do autor(a), em alguns casos, a coincidência das iniciais não garantiam a identificação, estes casos foram classificados como "indefinidos". O mesmo método foi utilizado para os prenomes estrangeiros. Quanto ao problema da coautoria, como este estudo tem como objetivo mensurar a atividade científica das mulheres e homens, foram computados os autores e co-autores de cada artigo. Quando não foi possível identificar o sexo pelo prenome/sobrenome foi utilizada a classificação alternativa de "indefinido".

A definição de sexo para fazer a classificação da produção científica analisada neste artigo foi bastante satisfatória para as áreas de conhecimento das Ciências Humanas, Sociais Aplicadas, 
Lingüísticas, Letras e Artes, onde apenas em média $1 \%$ dos(as) autores(as) não teve a variável sexo definida. Isso provavelmente deve-se a tradição dessas áreas de conhecimento, nas quais a produção científica é assinada com prenome e sobrenome. Nas áreas das Ciências da Saúde, Agrária, Biológicas, Exatas e da Terra a média de indefinidos segundo o sexo ficou em torno de $10 \%$, percentual que ainda pode ser considerado como um bom resultado. O drama ficou com as Engenharias, onde a média de "indefinidos" foi de cerca de $50 \%$. Esta área mantém a tradição de referenciar as pessoas pelo sobrenome e a assinatura da produção científica da área segue fortemente essa tradição.

Com objetivo de criar dois indicadores de avaliação: medida de atividade e medida de colaboração por gênero da produção científica nacional, este estudo utiliza a variável número de artigos publicados, identificados e classificados segundo o sexo dos(as) seus(uas) autores(as), por área de conhecimento $e$ ano de publicação. Os indicadores apresentam os dados quantitativos sobre as publicações brasileiras indexadas na base do SciELO, esses dados não expressam qualidade, mas a quantidade de resultado obtido pela pesquisa nacional, bem como a colaboração existente no meio científico. São, portanto, medidas estimadas da atividade científica do país $\mathrm{E}$, no caso particular do objetivo deste artigo, possibilitará pela primeira vez uma avaliação quantitativa $e$ de colaboração da produção científica feminina.

\section{A produção científica no SciELO}

Um dos mais significativos estudos bibliométricos nacionais, realizado por De Méis (1996), utilizou a base de dados do ISI e concluiu que a ciência brasileira no cenário internacional cresceu significativamente desde os anos 1980, atingindo, em 2001, 1,44\% do total da produção dessa base de dados. (Leta \& Cruz, 2003:137) As publicações citadas acima avaliam esta produção do ponto de vista da sua repercussão internacional, mas este trabalho limita-se a analisar a base de dados brasileira que se tornou disponível a 
A produção científica brasileira no feminino

partir de 1997, indexando as publicações brasileiras, incluindo as ciências humanas e sociais. Ainda é restrito o número das publicações nacionais catalogadas, embora crescentes ao longo desses oito anos. Vejam sua evolução nos comentários abaixo.

A tabela 1 mostra a evolução do número de artigos catalogados na base de dados do SciELO segundo as áreas científicas. A ampliação dessa base de dados no período estudado foi significativa e seguramente expressa os avanços da ciência e da tecnologia no Brasil. Atualmente, a base acumula um total de 56.694 artigos indexados, com 195.151 autores e co-autores. O crescimento dessa base de dados foi de $19,96 \%$ ao ano em média entre 1997 e 2005. Esses números retratam o desenvolvimento científico nacional via institutos de pesquisa e a multiplicação dos programas de pós-graduação na última década.

Os dados da tabela citada mostram que as áreas têm produções distintas; estas dependem em outras coisas do número de pessoas que trabalham nessas atividades de pesquisas bem como do número de periódicos indexados em cada uma. A concentração de periódicos na área da saúde é enorme: observase que em 1997 a participação dessa área era de 55\% dos artigos catalogados na base e em 2005 esta taxa caiu dez pontos percentuais, mas ainda assim foi de $44 \%$ sua participação no SciELO. Mantém-se na liderança e é a área de maior peso na biblioteca. Provavelmente isso se deva mais ao peso da participação das revistas indexadas da área de saúde, do que propriamente a uma maior produtividade dos pesquisadores(as). ${ }^{9}$ Isso também pode ser uma parte da explicação de porque as ciências agrárias, que detinham 8,6\% em 1997, passam para 19,8\% em 2005, com a taxa de crescimento mais expressiva da base $32,6 \%$ ao ano em média no período considerado. Em termos de crescimento destacam-se ainda as ciências biológicas, exatas e da

9 O peso da área de Ciências da Saúde seguramente deve-se a forma como esta biblioteca foi organizada numa parceria entre a BIREME e a FAPESP que incorporava a indexação da primeira biblioteca inteiramente dedicada a área da saúde. 
terra e engenharias, todas com taxas superiores a $20 \%$ nesses anos. No mínimo os cientistas dessas áreas fizeram um esforço especial para indexar os seus periódicos e aumentar o prestígio do seu campo disciplinar.

Por sua vez as ciências humanas, que em 1997 detinha $14,5 \%$ de participação e era a segunda no ranking, está na terceira posição e sua taxa de participação caiu para 10,7\% em 2005 . Notem que em 1997 o SciELO já tinha uma grande predominância das ciências da saúde e esta manteve-se ao longo dos anos. Essas informações podem ser visualizadas no gráfico 1. Dessa maneira, o SciELO apresenta um viés para as ciências ditas "duras", apesar de apresentar um esforço de indexação na área de Ciências Humanas.

Como foi observado acima a produção científica presente na SciELO não corresponde a produção científica nacional por área do conhecimento, na medida em que o número de periódicos indexados na base define a participação de cada uma dessas áreas de conhecimento na biblioteca eletrônica. Por exemplo, a área ciências da saúde apresenta metade dos artigos disponíveis nesta base: como no momento da organização do SciELO os(as) pesquisadores(as) submeteram um número maior de periódicos anteriores a 1997 para serem examinados pelo comitê do SciELO, isso marcou a presença da Saúde na base (tabela 1). De forma semelhante agiram as ciências agrárias $e$, atualmente, os periódicos dessa área de conhecimento significam $20 \%$ dos artigos indexados na biblioteca. Ciências Biológicas, Exatas e Humanas apresentam uma participação semelhante na base, em torno de $10 \%$ da produção indexada. As Engenharias participam com cerca de $4 \%$ dos artigos publicados, e as Ciências Sociais e Lingüística participam marginalmente no SciELO. Esses dados sobre produção científica refletem a distribuição assimétrica dos periódicos indexados pelas áreas do conhecimento, e não da distribuição dos recursos humanos pelas áreas ou de maior produtividade dessa ou daquela área (tabela 1 e gráfico 1 ). 
A produção científica brasileira no feminino

Tabela 1

Brasil, Números de Artigos Indexados no SciELO por Área do Conhecimento - (1985-2005).

\begin{tabular}{|c|c|c|c|c|c|c|c|c|c|}
\hline ano & $\begin{array}{l}\text { Ciências da } \\
\text { Saúde }\end{array}$ & $\begin{array}{l}\text { Ciências } \\
\text { Agrárias }\end{array}$ & $\begin{array}{c}\text { Ciências } \\
\text { Biológicas }\end{array}$ & $\begin{array}{l}\text { C. Exatas \& } \\
\text { da Terra }\end{array}$ & $\begin{array}{l}\text { Ciências } \\
\text { Humanas }\end{array}$ & $\begin{array}{l}\text { Engenha- } \\
\text { rias }\end{array}$ & $\begin{array}{l}\text { C. Sociais } \\
\text { Aplicadas }\end{array}$ & $\begin{array}{l}\text { Linguíst. L\& } \\
\text { Art. }\end{array}$ & total \\
\hline 1985 & 18 & - & - & - & - & - & - & - & 18 \\
\hline 1986 & 58 & - & - & - & - & - & - & - & 58 \\
\hline 1987 & 59 & - & - & - & - & - & - & - & 59 \\
\hline 1988 & 99 & - & - & - & - & - & - & - & 99 \\
\hline 1989 & 111 & - & - & - & - & - & - & - & 111 \\
\hline 1990 & 114 & - & - & - & - & - & - & - & 114 \\
\hline 1991 & 117 & - & - & - & - & - & - & - & 117 \\
\hline 1992 & 128 & 23 & - & - & - & - & - & - & 151 \\
\hline 1993 & 157 & 72 & - & - & - & - & - & - & 229 \\
\hline 1994 & 173 & 89 & - & - & - & - & - & - & 262 \\
\hline 1995 & 172 & 94 & 19 & & 37 & - & - & - & 322 \\
\hline 1996 & 166 & 56 & 24 & - & 65 & - & - & - & 311 \\
\hline 1997 & 1102 & 172 & 144 & 155 & 289 & 63 & 47 & 19 & 1991 \\
\hline 1998 & 1521 & 309 & 203 & 336 & 425 & 73 & 49 & 21 & 2937 \\
\hline 1999 & 1673 & 472 & 322 & 464 & 400 & 155 & 73 & 27 & 3586 \\
\hline 2000 & 2114 & 1037 & 425 & 573 & 498 & 245 & 103 & 25 & 5020 \\
\hline 2001 & 2516 & 1317 & 598 & 521 & 743 & 257 & 103 & 20 & 6075 \\
\hline 2002 & 3228 & 1687 & 634 & 801 & 808 & 313 & 130 & 15 & 7616 \\
\hline 2003 & 3871 & 1994 & 866 & 679 & 941 & 347 & 148 & 56 & 8902 \\
\hline 2004 & 4231 & 2225 & 1049 & 1035 & 980 & 385 & 176 & 53 & 10134 \\
\hline 2005 & 3957 & 1645 & 906 & 825 & 814 & 290 & 78 & 23 & 8538 \\
\hline 2006 & 44 & - & - & - & - & - & - & - & 44 \\
\hline total & 25629 & 11192 & 5190 & 5389 & 6000 & 2128 & 907 & 259 & 56694 \\
\hline $\begin{array}{c}\text { cresc. a.a. } \\
\text { (1997-2004) }\end{array}$ & $17,00 \%$ & $33,00 \%$ & $26,00 \%$ & $23,00 \%$ & $14,00 \%$ & $21,00 \%$ & $7,00 \%$ & $2,00 \%$ & $20,00 \%$ \\
\hline
\end{tabular}

* em 2006 foi computado até janeiro.

A taxa de crescimento foi calculada pela média geométrica.

A SciELO entrou em funcionamento á partir de 1997, dessa forma, a produção científica anterior a 1997 se refere a periódicos disponibilizados no momento da organização da biblioteca. 
Gráfico 1

Produção Científica Indexada na SciELO por Área do Conhecimento

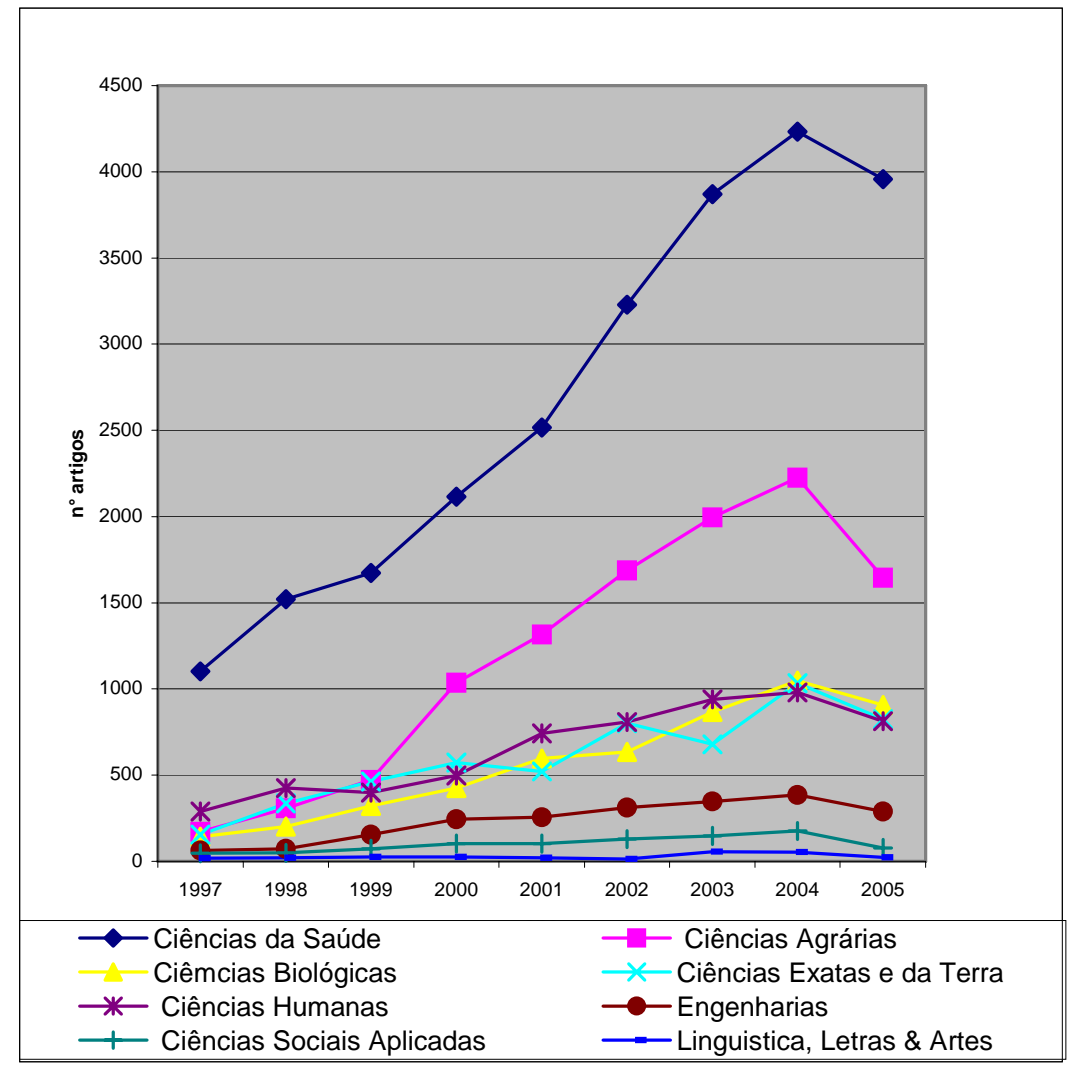

Fonte: Scielo (http://www.scielo.br), elaboração própria.

Nesses anos de desenvolvimento, este banco de dados cresceu em torno de 19,96\% ao ano; algumas áreas tiveram taxas superiores: as Ciências Agrárias e a Biológica apresentaram o maior ritmo de crescimento no processo de indexação de seus periódicos, $33 \%$ e $26 \%$ em média ao ano respectivamente. Isso demonstra um esforço dos(as) pesquisadores(as) dessas áreas para 
A produção científica brasileira no feminino

aumentar a disseminação e visibilidade da literatura científica pertinente a essas áreas do conhecimento. Logo abaixo, mas na média, ficaram Ciências Exatas, Engenharias e Ciências da Saúde, todas com um crescimento em torno de $20 \%$ ao ano em média. Enquanto que Ciências Humanas (14\% a.a.), Ciências Sociais (7\% a.a.) e Lingüística $(2 \%$ a.a.) apresentaram crescimento inferior à média da expansão do SciELO. Portanto, nota-se um esforço das chamadas "ciências duras" para estarem presentes na biblioteca eletrônica, divulgando e disseminando sua produção. Talvez o fato de que os cientistas dessas áreas têm uma grande tradição no desenvolvimento do sistema científico e tecnológico do Brasil, desde o final dos anos 1940, confira a esses setores maior maturidade científica do que as humanas e sociais, de desenvolvimento mais recente.

Para complementar as informações gerais a respeito da base de dados SciELO buscou-se a origem das instituições responsáveis pelas publicações indexadas na base (tabelas 2, 3 e 4). Neste trabalho foram analisados 195.151 autores(as), e o número de artigos encontrados na base foi de 56.694. A primeira grande constatação é que a pesquisa brasileira processa-se nas universidades públicas e nos institutos de pesquisa (públicos): as Instituições de Ensino Superior (IES) públicas são responsáveis por $54 \%$ dos periódicos indexados no SciELO.

Pode-se concluir que o peso do Estado é enorme no avanço e divulgação da ciência e da tecnologia nacional e a enorme concentração no estado de São Paulo expressa a íntima relação entre desenvolvimento econômico e a produção científica $e$ tecnológica. Pois, o estado que concentra o Produto Interno Bruto (PIB) nacional também domina o espaço científico brasileiro, como é o caso do estado paulista e fluminense que juntos respondem por $76,19 \%$ dos periódicos indexados (tabelas 3 e 4).

Uma última observação que suscita a base de dados SciELO refere-se ao número expressivo de autores encontrado no período analisado. A grande conclusão é que a colaboração é uma prática científica comum no mundo científico e o Brasil não foge a regra, 
a média encontrada da base de dados (SciELO) foi 3,4 autores(as) por artigo. No entanto, é preciso esclarecer que não foi considerado na caso da co-autoria a importância dessa atividade e não se analisou se a colaboração foi realizada no âmbito da mesma instituição ou de organizações diferentes ou com pesquisadores estrangeiros.

Tabela 2

Natureza das Instituições Responsáveis pela Publicação dos Periódicos Indexados na SciELO, 1997/2005

\begin{tabular}{l|c|c|c|c}
\hline \multirow{2}{*}{ instiuições } & \multicolumn{2}{|c|}{ artigos } & \multicolumn{2}{c}{ periódicos } \\
\cline { 2 - 5 } & $\mathbf{n}^{\mathbf{0}}$ & $\mathbf{\%}$ & $\mathbf{n}^{\mathbf{0}}$ & $\mathbf{\%}$ \\
\hline IES Publicas & 27963 & 49,32 & 79 & 53,74 \\
IES Privadas & 692 & 1,22 & 6 & 4,08 \\
institutos de pesquisa & 10388 & 18,32 & 21 & 14,29 \\
associações e sociedades ciêntíficas & 17651 & 31,13 & 41 & 27,89 \\
total & $\mathbf{5 6 6 9 4}$ & $\mathbf{1 0 0}$ & $\mathbf{1 4 7}$ & $\mathbf{1 0 0}$ \\
\hline
\end{tabular}

Nota: Os artigos são referentes ao total de artigos publicados nos periódicos, devendo ser interpretados como volume de informação de um periódico e abrange os periódicos publicados de 1997/2005.

Fonte: Scielo (http://www.scielo.br), elaboração própria.

Gráfico 2

Natureza das Instituições Responsáveis pela publicação dos Periódicos Indexados na SciELO

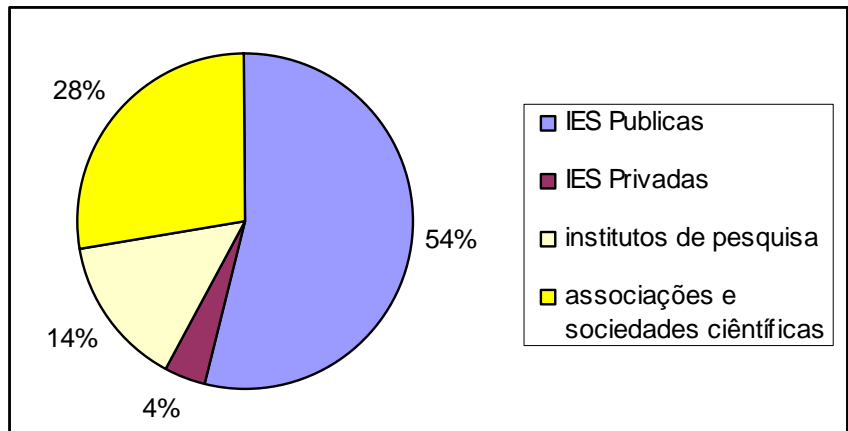

Fonte: Scielo (http://www.scielo.br), elaboração própria. 
A produção científica brasileira no feminino

Tabela 3

Instituições com maior Participação nos Periódicos Indexados no SciELO

\begin{tabular}{l|c|c}
\hline \multirow{2}{*}{ Instituição } & \multicolumn{2}{|c}{ periódicos } \\
\cline { 2 - 3 } & $\mathbf{n}^{\mathbf{0}}$ & $\%$ \\
\hline USP & 32 & 45,07 \\
UNICAMP & 10 & 14,08 \\
UNESP & 7 & 9,86 \\
UFRGS & 4 & 5,63 \\
FIOCRUZ & 4 & 5,63 \\
UFSCar & 3 & 4,23 \\
UFRJ & 3 & 4,23 \\
UFPR & 3 & 4,23 \\
UFV & 3 & 4,23 \\
UFSM & 2 & 2,82 \\
total & $\mathbf{7 1}$ & $\mathbf{1 0 0}$
\end{tabular}

Fonte: Scielo (http://www.scielo.br), elaboração própria.

Tabela 4

Periódicos Indexados no SciELO, segundo unidades da federação (\%)

\begin{tabular}{l|c|c}
\hline \multirow{2}{*}{ estado da publicação } & \multicolumn{2}{|c}{ periódicos } \\
\cline { 2 - 3 } & $\mathbf{n}^{\circ}$ & $\%$ \\
\hline AM & 1 & 0,68 \\
BA & 1 & 0,68 \\
DF & 7 & 4,76 \\
MG & 6 & 4,08 \\
PB & 1 & 0,68 \\
PE & 1 & 0,68 \\
PR & 5 & 3,4 \\
RJ & 29 & 19,73 \\
RN & 1 & 0,68 \\
RS & 11 & 7,48 \\
SC & 1 & 0,68 \\
SP & 83 & 56,46 \\
total & $\mathbf{1 4 7}$ & $\mathbf{1 0 0}$ \\
\hline
\end{tabular}

Fonte: Scielo (http://www.scielo.br), elaboração própria. 
Hildete Melo e André Oliveira

\section{Medida de atividade: gênero $e$ a produção científica}

No item anterior apresenta-se um quadro geral da base de dados SciELO, agora vamos procurar as mulheres engajadas no sistema científico e tecnológico brasileiro. Essa busca implica em reforçar a idéia de que só o olhar interessado de pesquisadoras(es) envolvidas(os) com a questão de gênero pode encontrar a mulher, onde as estatísticas friamente tratam igualmente os desiguais. Isso apesar do consenso atual de que a perspectiva de gênero contribui positivamente para apontar as diferenças discriminatórias dos papéis sociais.

Para avaliar a produção científica nacional fez-se uma breve análise sobre a presença de mulheres e homens no Diretório dos Grupos de Pesquisa do CNPq para o período 2000/2006, complementando trabalho anterior de Melo e Lastres (2004) sobre a presença feminina no sistema científico e tecnológico nacional na década de 1990. Carneiro Jr \& Lourenço (2003) apontam que em 2002 essa base de dados abrigava mais de 230 mil currículos de pesquisadores, docentes, tecnólogos e estudantes e abrangia $85 \%$ dos pesquisadores brasileiros. ${ }^{10}$ Acreditamos que esta base de dados é uma fonte importante de informações sobre as atividades de pesquisa realizadas no país.

As tabelas $5 \mathrm{a}$ e $5 \mathrm{~b}$ apresentam a distribuição dos(as) pesquisadores(as) cadastrados(as) no Diretório dos Grupos de Pesquisa do $\mathrm{CNPq}$ distribuídos pelas áreas do conhecimento e sexo. Como a década de noventa já foi analisada por Melo \& Lastres (2006), escolheu-se os anos de 2002 e $2005^{11}$, porque estes são os anos finais do banco de dados SciELO e pode-se ter um panorama relativo do meio científico brasileiro do ponto de vista do sexo dos seus participantes. Houve um crescimento

${ }^{10} \mathrm{O}$ acesso a esta base de dados é feito pela internet: http://www.cnpq.br, PlataformaLattes, Diretório dos Grupos de Pesquisa e Currículos Lattes.

${ }^{11}$ Os dados do ano de 2005 são oriundos da base de dados do CNPq, e foram coletados entre janeiro e abril de 2006, optou-se então defini-los como referentes ao ano de 2005. 
A produção científica brasileira no feminino

dos pesquisadores nacionais de cerca de $1,4 \%$ no período, mas as mulheres tiveram um taxa de crescimento de $1,4 \%$ (na média) $e$ os homens de $1,36 \%$, isso provavelmente mostra um pequeno empoderamento feminino no sistema científico brasileiro, refletindo o melhor desempenho no ensino superior. As taxas de crescimento são muito próximas, o diferencial feminino talvez seja a maciça presença das mulheres nas áreas de saúde $e$ humanas, que são as de maior participação no conjunto dos(as) pesquisadores(as). Notem que as áreas de Lingüística e Ciências Humanas têm cerca de $60 \%$ dos seus pesquisadores do sexo feminino e as áreas de Ciências da Saúde e Biológicas concentram mais de $50 \%$ de mulheres. Portanto, das oito áreas do conhecimento listadas, em metade delas a participação feminina é significativa.

As mulheres aproveitaram bem a revolução provocada pelo movimento feminista nos anos sessenta e setenta do século XX e a expansão da pós-graduação brasileira nos últimos vinte anos, passando a valorizar a carreira profissional. ${ }^{12}$ No entanto, mulheres e homens não fazem a mesma trajetória do ponto de vista das carreiras científicas. $\mathrm{O}$ sexo feminino ainda permanece marcado pelo estereótipo do papel dos "cuidados", escolhem as áreas vinculadas à educação, saúde e a assistência social. Os homens, seguindo no rastro do papel definido socialmente para o sexo masculino, buscam a aventura do descobrimento dos campos científicos como a engenharia, ciências exatas e da terra e as agrárias. Eles também são aprisionados no seu papel, mas numa concentração inferior a encontrada para as mulheres. Estas são em torno de $44 \%$ das pesquisadoras apenas nas áreas das ciências humanas e da saúde. Por sua vez, eles em engenharia $e$ ciências exatas e da terra são aproximadamente $35 \%$ do total de pesquisadores (tabelas $5 a$ e $5 b$ ).

${ }^{12}$ Sobre o assunto, ver Bruschini e Lombardi, 1999 e Lombardi, 2004. 
Hildete Melo e André Oliveira

Tabela 5 (a)

Brasil, número de pesquisadores e sexo, segundo área do conhecimento do grupo de pesquisa do $\mathrm{CNPq}(\%)$

\begin{tabular}{l|c|c|c|c|c|c}
\hline \multirow{2}{*}{ Área do Conhecimento } & \multicolumn{3}{|c|}{$\mathbf{2 0 0 2}$} & \multicolumn{3}{c}{ 2005/04-2006* } \\
\cline { 2 - 7 } & total & masc. & fem. & total & masc. & fem. \\
\hline Ciências Agrárias & 12,02 & 14,91 & 8,57 & 11,17 & 13,71 & 8,32 \\
Ciências Biológicas & 12,93 & 11,30 & 14,88 & 12,09 & 10,72 & 13,73 \\
Ciências da Saúde & 16,43 & 12,64 & 20,96 & 17,59 & 13,54 & 22,15 \\
Ciências Exatas e da Terra & 12,53 & 15,93 & 8,46 & 11,60 & 14,83 & 7,92 \\
Ciências Humanas & 17,07 & 12,47 & 22,55 & 17,15 & 12,75 & 22,15 \\
Ciências Sociais Aplicadas & 9,22 & 8,92 & 9,59 & 10,80 & 10,83 & 10,71 \\
Engenharias & 15,26 & 21,18 & 8,20 & 14,78 & 20,63 & 8,11 \\
Lingüística, Letras e Artes & 4,54 & 2,65 & 6,80 & 4,83 & 3,00 & 6,92 \\
total (100\%) & $\mathbf{6 3 3 4 2}$ & $\mathbf{3 4 4 6 7}$ & $\mathbf{2 8 8 6 5}$ & $\mathbf{8 8 2 5 5}$ & $\mathbf{4 6 9 6 5}$ & $\mathbf{4 0 8 6 5}$ \\
\hline
\end{tabular}

Fonte: Diretório dos Grupos de Pesquisa, CNPq, 2006 (http://dgp. cnpq.br/planotabular/).

Tabela 5 (b)

Brasil, número de pesquisadores e sexo, segundo área do conhecimento do grupo de pesquisa (\%)

\begin{tabular}{l|c|c|c|c|c|c}
\hline \multirow{2}{*}{ Área do Conhecimento } & \multicolumn{3}{|c|}{$\mathbf{2 0 0 2}$} & \multicolumn{3}{|c}{ 2005/04-2006* } \\
\cline { 2 - 7 } & total & masc. & fem. & total & masc. & fem. \\
\hline Ciências Agrárias & 100 & 67,51 & 32,49 & 100 & 65,34 & 34,48 \\
Ciências Biológicas & 100 & 47,55 & 52,44 & 100 & 47,18 & 52,59 \\
Ciências da Saúde & 100 & 41,86 & 58,13 & 100 & 40,95 & 58,32 \\
Ciências Exatas e da Terra & 100 & 69,20 & 30,77 & 100 & 68,06 & 31,64 \\
Ciências Humanas & 100 & 39,77 & 60,20 & 100 & 39,55 & 59,81 \\
Ciências Sociais Aplicadas & 100 & 52,61 & 47,36 & 100 & 53,34 & 45,88 \\
Engenharias & 100 & 75,51 & 24,49 & 100 & 74,30 & 25,41 \\
Lingüística, Letras e Artes & 100 & 31,73 & 68,27 & 100 & 33,04 & 66,26 \\
total & $\mathbf{1 0 0}$ & $\mathbf{5 4 , 4 1}$ & $\mathbf{4 5 , 5 7}$ & $\mathbf{1 0 0}$ & $\mathbf{5 3 , 2 2}$ & $\mathbf{4 6 , 3}$ \\
\hline
\end{tabular}

Fonte: Diretório dos Grupos de Pesquisa, CNPq, 2006 (http: //dgp. cnpq.br/planotabular/).

Nota: O total inclui pesquisadores cujo sexo não foi identificado.

*os dados de 2006 se referem à base atualizada em 01/04/2006. 
A produção científica brasileira no feminino

A evolução da produção científica indexada na base de dados do SciELO e discriminada pelas áreas do conhecimento analisada neste trabalho, e 1997 havia um total de 1991 artigos indexados na base e no final de 2005 a base acumulava 56.694 artigos. Notem que foi uma expansão significativa de aproximadamente $19,96 \%$ a.a. em média nesses anos. Segundo Leta e Cruz (2003), nessa avaliação deve-se levar em consideração que as áreas do conhecimento têm produtividades diferentes, em parte dependente do número de pesquisadores trabalhando em cada área.

As tabelas $6 \mathrm{a}$ e $6 \mathrm{~b}$ mostram a produção científica brasileira segundo o número de autores e co-autores dos artigos publicados na base de dados do SciELO e área do conhecimento. O método utilizado para identificar o sexo dos autores permitiu definir o sexo de $82,85 \%$ dos autores da base do SciELO: o que para o programa de computador adotado revelou-se bastante satisfatório.

A análise iniciou-se pela distribuição dos autores pelas diversas áreas do conhecimento, notem que permanece a distorção dada pelo o número diferenciado de revistas indexadas na base de dados. Isso pode levar a uma falsa conclusão de que esta ou aquela área tenha maior ou menor produtividade, claro que a indexação define um comportamento mais ou menos agressivo de uma área em relação às outras, bem como recursos mais abundantes que permitem manter um programa de publicação efetivo. A taxa de participação das publicações relativas às Ciências da Saúde representa $50.35 \%$ dos autores citados no SciELO. O número de revistas contido nessa base explica, claramente, a extraordinária taxa de participação, que vai muito além da participação dos pesquisadores no diretório dos Grupos de Pesquisa do CNPq. O mesmo acontece nas Ciências Agrárias com $23,8 \%$ de taxa de participação e praticamente $75 \%$ da produção científica nacional. Isso expressa a distorção do peso da indexação das ciências da saúde e agrárias na base de dados do SciELO. 
Passando para um olhar de gênero, na Tabela 6a nota-se que as mulheres identificadas representam uma taxa de participação de $32,28 \%$ dos autores. De forma interessante esta taxa é quase similar à encontrada por Melo e Lastres (2006) na análise das bolsistas de produtividade do $\mathrm{CNPq}$ para a década de noventa do século $\mathrm{XX}$, o que relaciona a produção de conhecimentos com pesquisadoras(es) realmente engajados em atividades científicas. A participação das mulheres, comparada aos homens, aponta para uma concentração da produção nas Ciências Humanas e Lingüística, Letras e Artes.

Tabela 6 (a)

Total da Produção Científica SciELO por Sexo e Área do Conhecimento

\begin{tabular}{l|c|c|c|c|c|c|c|c}
\hline & \multicolumn{4}{|c|}{ sexo } & \multicolumn{4}{c}{$\%$} \\
\cline { 2 - 9 } Área do Conhecimento & masc. & fem. & indef. & total & masc. & fem. & indef. & total \\
\hline Ciências da Saúde & 48285 & 35980 & 14012 & 98277 & 49,13 & 36,61 & 14,26 & 100 \\
Ciências Agrárias & 27430 & 11081 & 8008 & 46519 & 58,97 & 23,82 & 17,21 & 100 \\
Ciências Biológicas & 7117 & 5636 & 3348 & 16101 & 44,2 & 35 & 20,79 & 100 \\
Ciências Exatas e da Terra & 8200 & 4325 & 4553 & 17078 & 48,01 & 25,32 & 26,66 & 100 \\
Ciências Humanas & 3944 & 4484 & 90 & 8518 & 46,3 & 52,64 & 1,06 & 100 \\
Engenharias & 2363 & 668 & 3826 & 6857 & 34,46 & 9,74 & 55,8 & 100 \\
Ciências Sociais Aplicadas & 830 & 665 & 18 & 1513 & 54,86 & 43,95 & 1,19 & 100 \\
Linguística, Letras e Artes & 133 & 150 & 5 & 288 & 46,18 & 52,08 & 1,74 & 100 \\
total & $\mathbf{9 8 3 0 2}$ & $\mathbf{6 2 9 8 9}$ & $\mathbf{3 3 8 6 0}$ & $\mathbf{1 9 5 1 5 1}$ & $\mathbf{5 0 , 3 7}$ & $\mathbf{3 2 , 2 8}$ & $\mathbf{1 7 , 3 5}$ & $\mathbf{1 0 0}$ \\
\hline
\end{tabular}

Fonte: SciELO (http://www.scielo.br), elaboração própria.

A menor participação feminina é na área das Engenharias, o que não deixa de ser uma conclusão esperada. Afinal, as mulheres ainda não escolhem as carreiras das Engenharias como profissão $e$, dessa forma, há menos pesquisas realizadas por mulheres nesse campo científico. Embora a análise da Engenharia tenha ficado em parte prejudicada pelo grande número de autores não identificáveis como foi explicitado no item metodológico (Gráfico 3), a análise do diretório dos Grupos de Pesquisa do CNPq indica que essa participação ainda é baixa nessa carreira.

$\mathrm{O}$ viés que se origina pela alta participação na base de dados SciELO da indexação na área das ciências da saúde, mostra que esta área concentra tanto os homens como as mulheres, há 
A produção científica brasileira no feminino

um equilíbrio de ambos os sexos na busca dessa carreira científica, embora o comportamento feminino, como mostra o Gráfico 3, apresente-se de forma mais contundente. Essa trajetória indica que as mulheres caminham para uma grande presença científica nessa área como atestam as informações desse banco de dados.

Tabela 6 (b)

Total da Produção Científica SciELO por Sexo e Área do Conhecimento, considerando apenas os Identificados por Sexo

\begin{tabular}{l|c|c|c|c}
\hline \multirow{2}{*}{ Área do Conhecimento } & \multicolumn{2}{|c|}{ total fe m. = 100\% } & \multicolumn{2}{c}{ total * = 100\% } \\
\cline { 2 - 5 } & masc. & fem. & masc. & fem. \\
\hline Ciências da Saúde & 49,12 & 57,12 & 57,3 & 42,7 \\
Ciências Agrárias & 27,9 & 17,59 & 71,23 & 28,77 \\
Ciências Biológicas & 7,24 & 8,95 & 55,81 & 44,19 \\
Ciências Exatas e da Terra & 8,34 & 6,87 & 65,47 & 34,53 \\
Ciências Humanas & 4,01 & 7,12 & 46,8 & 53,2 \\
Engenharias & 2,4 & 1,06 & 77,96 & 22,04 \\
Ciências Sociais Aplicadas & 0,84 & 1,06 & 55,52 & 44,48 \\
Linguística, Letras e Artes & 0,14 & 0,24 & 47 & 53 \\
total & $\mathbf{1 0 0}$ & $\mathbf{1 0 0}$ & $\mathbf{6 0 , 9 5}$ & $\mathbf{3 9 , 0 5}$ \\
\hline
\end{tabular}

Nota:*o total apresenta apenas os autores que foram identificados por sexo, os indefinidos não constam.

Fonte: SciELO (http://www.scielo.br), elaboração própria.

Gráfico 3

Participação Feminina por Área do Conhecimento na Produção Científica SciELO

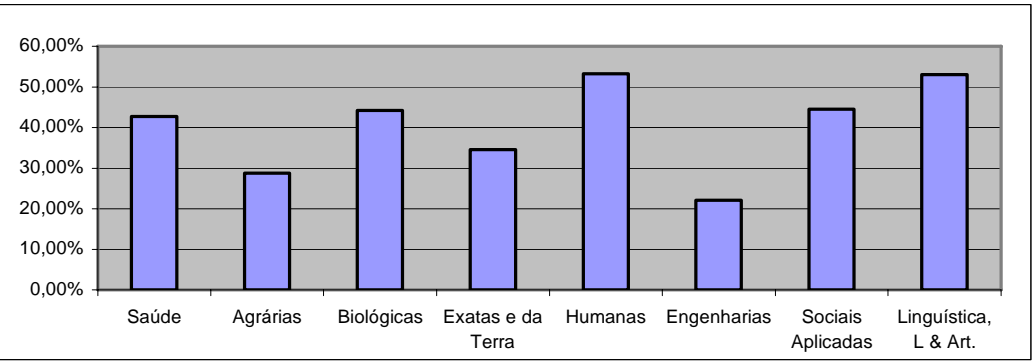

Nota: a porcentagem foi calculada com o total excluindo os indefinidos Fonte: SciELO (http://www.scielo.br), elaboração própria. 
Hildete Melo e André Oliveira

Tabela 7

Participação Feminina Efetiva na Produção Científica por Área do Conhecimento (\%)

\begin{tabular}{lccccccccc} 
Áreas do Conhecimento & $\mathbf{1 9 9 7}$ & $\mathbf{1 9 9 8}$ & $\mathbf{1 9 9 9}$ & $\mathbf{2 0 0 0}$ & $\mathbf{2 0 0 1}$ & $\mathbf{2 0 0 2}$ & $\mathbf{2 0 0 3}$ & $\mathbf{2 0 0 4}$ & $\mathbf{2 0 0 5}$ \\
\hline Ciências da Saúde & 0,44 & 0,43 & 0,43 & 0,41 & 0,41 & 0,43 & 0,42 & 0,43 & 0,43 \\
Ciências Agrárias & 0,31 & 0,35 & 0,29 & 0,27 & 0,27 & 0,27 & 0,29 & 0,30 & 0,30 \\
Ciências Biológicas & 0,44 & 0,46 & 0,52 & 0,48 & 0,42 & 0,43 & 0,45 & 0,44 & 0,42 \\
Ciências Exatas e da Terra & 0,24 & 0,31 & 0,34 & 0,36 & 0,33 & 0,35 & 0,34 & 0,36 & 0,35 \\
Ciências Humanas & 0,46 & 0,51 & 0,53 & 0,52 & 0,51 & 0,56 & 0,53 & 0,56 & 0,56 \\
Engenharias & 0,06 & 0,17 & 0,19 & 0,16 & 0,18 & 0,16 & 0,24 & 0,23 & 0,31 \\
Ciências Sociais Aplicadas & 0,66 & 0,61 & 0,63 & 0,46 & 0,45 & 0,35 & 0,44 & 0,42 & 0,29 \\
Linguistica, Letras e Artes & 0,65 & 0,63 & 0,45 & 0,57 & 0,67 & 0,67 & 0,46 & 0,49 & 0,43 \\
\hline
\end{tabular}

Nota: a participação feminina efetiva na produção científica foi obtida pela razão: número de autoras do sexo feminino em artigos na área do conhecimento i e no ano j/ número total de autores e autoras com sexo identificado na área do conhecimento i e no ano j.

Fonte: SciELO (http://www.scielo.br), elaboração própria.

A análise da evolução da participação feminina na produção científica ao longo do período considerado (tabela 7) indica uma redução da participação feminina na produção científica das Ciências Sociais e Lingüística ${ }^{13}$, um aumento nas Engenharias, Humanas e Exatas e uma certa estabilidade nas Ciências da Saúde, Agrárias e Biológicas. A maior variação em algumas áreas parece ser resultante da variação da amostra que, se no início é menor, vai se ampliando no decorrer do tempo, de forma que a participação nos últimos anos não se altera muito $e$ parece ser mais próxima da participação dos sexos na produção científica. A despeito disto, houve um aumento real da participação feminina nas Engenharias que, em 2005, foi de 31\%, maior que os $22 \%$ da análise estática, o mesmo acontecendo nas

${ }^{13}$ Talvez isso seja causado pela indexação de revistas ligadas a Economia, tais como: Revista Brasileira de Economia, Revista de Economia e Sociologia Rural e Economia Política com taxas de participação femininas relativamente baixas, que explica essa redução da participação feminina numa área tradicionalmente vinculada ao estereótipo do "ser mulher". 
A produção científica brasileira no feminino

Ciências Exatas e Humanas. Mesmo com a maior participação masculina na produção científica, pode-se concluir que nesses anos as mulheres estão aumentando sua participação no meio científico e tecnológico (Gráfico 4).

\section{Gráfico 4}

Evolução da Participação Feminina na Produção Científica por Área do Conhecimento (\%)

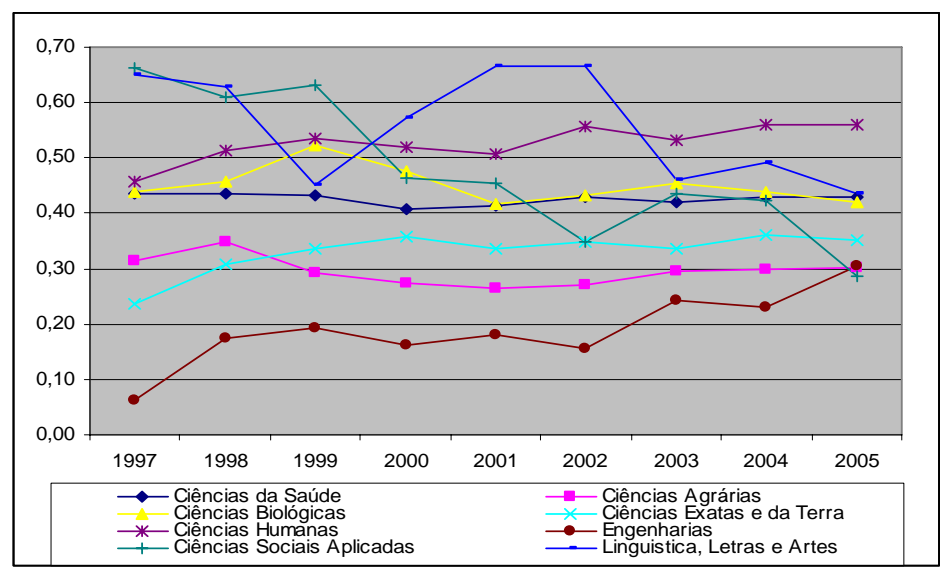

Por outro lado, há um aumento significativo da participação feminina nas áreas de ciências exatas e da terra e mesmo nas engenharias. Nessa última, talvez o fato de que foi possível identificar o sexo de um maior número de autores tenha contribuído para isso ou esse crescimento expressa as mudanças no mercado de trabalho e novas atitudes da sociedade em relação ao papel feminino. Nos anos 1990 amplia-se o emprego feminino e na mesma trajetória também os postos de trabalho de engenheiros(as), sobretudo para engenheiro químico $e$ de organização e métodos. Mesmo na engenharia, verifica-se que as mulheres também escolhem especializações mais próximas do estereótipo feminino e submetidas a "segregação horizontal" dentro da carreira (Lombardi, 2004). No caso das ciências exatas e 
da terra, provavelmente, a inclusão de periódicos da área de química tenha contribuído para este crescimento, como mostram as taxas de participação femininas das revistas Polímeros, Química Nova e Journal of the Brazilian Chemical Society.

\section{Medidas de colaboração, segundo gênero}

O número de artigos nas publicações indexadas com coautoria indica o grau de colaboração científica, mesmo que seja entre pessoas da mesma instituição. Nessa avaliação não foi possível estabelecer a instituição de origem dos(as) autores(as) dos artigos constantes da base de dados. A colaboração científica é um processo fundamental para o desenvolvimento científico, embora essa colaboração dependa das características de cada disciplina científica. Na tabela 8 foi construído o indicador de colaboração para a base de dados analisada e nota-se uma intensa rede de colaboração entre os(as) pesquisadores(as) das áreas de ciências agrárias, engenharias, exatas, biológicas e da saúde. Mesmo internacionalmente esta é uma tendência observada também nos demais centros de pesquisa. Provavelmente a colaboração nessas áreas seja uma forma de redução de custos, devido aos elevados preços dos equipamentos e técnicas que incentivam a colaboração.

As ciências sociais e humanas têm uma taxa de participação menor dos trabalhos com co-autoria, talvez por que nesse campo haja uma maior possibilidade de realizar pesquisas isoladas com baixo custo. Lingüística, Letras e Artes cujo índice de publicação de um só autor é de $84 \%$, essa taxa expressa bem a tendência das humanas e sociais de pesquisas solitárias.

Nas artes e humanidades, onde a participação feminina na produção científica é mais significativa, os homens participam mais da produção científica sem co-autoria do que as mulheres. Nas línguas, letras e artes a participação de homens e mulheres nas publicações sem co-autoras é similar para ambos, envolvendo em torno de $40 \%$ dos autores em relação a cada sexo nesta 
A produção científica brasileira no feminino

área, enquanto que as mulheres somam $10,42 \%$ e os homens $5,56 \%$ de co-autores nestas áreas, considerando o total de autores envolvidos. Nas ciências humanas, os homens têm maioria na produção científica em autoria única $(28,49 \%)$ e as mulheres têm maioria na forma de produção científica com co-autoria (28,45\%). Nas ciências sociais aplicadas os homens têm maioria tanto na produção científica com autoria única, $20,56 \%$ dos autores identificados e 34,30\% nos artigos com co-autorias da área.

Tabela 8

Medidas de colaboração por sexo e áreas do conhecimento na produção científica SciELO

\begin{tabular}{|c|c|c|c|c|c|c|c|}
\hline \multirow[b]{2}{*}{ áreas do conhecimento } & \multicolumn{3}{|c|}{ sem co-autoria } & \multicolumn{3}{|c|}{ com co-autoria } & \multirow{2}{*}{$\begin{array}{c}\text { total } \\
(100 \%)^{*}\end{array}$} \\
\hline & $\%$ masc. & \% fem. & $\%$ total $^{*}$ & $\%$ masc. & $\%$ fem. & $\%$ total $^{\star}$ & \\
\hline Linguística, Letras e Artes & 40,63 & 41,67 & 84,03 & 5,56 & 10,42 & 15,97 & 288 \\
\hline Ciências Sociais Aplicadas & 20,56 & 15,14 & 36,09 & 34,3 & 28,82 & 63,91 & 1513 \\
\hline Ciências Humanas & 28,49 & 24,2 & 53,25 & 17,81 & 28,45 & 46,75 & 8518 \\
\hline Ciências da Saúde & 2,91 & 1,48 & 4,71 & 46,23 & 35,13 & 95,29 & 98277 \\
\hline Ciências Biológicas & 1,94 & 0,82 & 4,06 & 42,26 & 34,18 & 95,94 & 16101 \\
\hline Ciências Exatas e da Terra & 3,63 & 0,72 & 5,98 & 44,38 & 24,6 & 94,02 & 17078 \\
\hline Engenharias & 1,74 & 0,2 & 2,58 & 32,73 & 9,54 & 97,42 & 6857 \\
\hline Ciências Agrárias & 0,45 & 0,08 & 0,64 & 58,51 & 23,75 & 99,36 & 46519 \\
\hline total & 3,57 & 2,14 & 6,2 & 46,8 & 30,14 & 93,8 & 195151 \\
\hline
\end{tabular}

Nota: *inclusive indefinidos.

Fonte: Scielo (http://www.scielo.br), elaboração própria.

Nas ciências chamadas "duras" os homens têm maioria na produção científica com autoria única e em co-autoria. Mais uma vez a participação feminina nesse campo científico confirma uma forma de trabalhar em cooperação das mulheres, a análise do SciELO mostra que a participação feminina é substancialmente na forma de co-autoria. A produção de artigos como autoria exclusiva não chega a $2 \%$ em nenhuma das áreas dessas ciências, considerando as publicações com autor único e com co-autoria em relação ao total das autorias das áreas, enquanto os homens têm no mínimo o dobro de autorias nesta forma de publicação. As mulheres aparecem como produtoras de ciências nessas áreas do conhecimento sempre na forma de co-autoria; é a consagração da 
Hildete Melo e André Oliveira

rede e da colaboração. Talvez as enormes dificuldades que as mulheres têm de conciliar a vida familiar e a dedicação exigida pela carreira científica impõe essa forma de trabalho.

\section{Considerações Finais}

Apesar de suas limitações, este trabalho não deixa dúvidas de que os dados coletados em bases como o SciELO são ferramentas importantes para avaliar o desempenho da atividade científica no país, sobretudo, ressaltando seu caráter pioneiro uma análise de gênero do sistema científico e tecnológico, propondo uma discussão sobre a sub-representação $e$ menor produtividade das mulheres nessas atividades. A base de dados SciELO é recente e espelha a necessidade das agências e dos órgãos nacionais em utilizar indicadores para decidir sobre a distribuição de recursos aos(as) pesquisadores(as), sejam bolsas ou financiamentos a projetos de pesquisa.

A metodologia utilizada para identificar o sexo dos autores e co-autores foi considerada bastante efetiva, porque apenas $17,3 \%$ destes autores tiveram o sexo indeterminado. Ainda assim, o grau de indeterminação foi diferente para as diversas áreas: expressivo nas engenharias $(50 \%)$ e praticamente inexistente em lingüística, ciências humanas e sociais (1\%); nas ciências da saúde, agrária, biológicas e exatas a indeterminação ficou em torno de $10 \%$. O protocolo científico de cada área aparentemente definiu o maior ou menor sucesso da aplicação desse método, como mostra o caso das Engenharias.

Nos últimos vinte anos a produção científica brasileira cresceu extraordinariamente, mas é uma produção concentrada na região Sudeste do Brasil, onde se localiza a maior parte das instituições universitárias, programas de pós-graduação e os(as) pesquisadores(as) nacionais. A análise constata que a pesquisa brasileira processa-se nas universidades públicas e nos institutos de pesquisa (públicos). 
A produção científica brasileira no feminino

A expansão da produção científica brasileira estimulou o surgimento de estudos sobre o tema, mas essa literatura não tem sido sensível a temática de gênero, o que confere um caráter pioneiro a esta análise a partir da construção dos indicadores: medida de atividade e medida de colaboração na perspectiva de gênero. De forma interessante, o sistema científico e tecnológico sob a aparente neutralidade da ciência ignora que mulheres $e$ homens têm trajetórias diferenciadas e sob esse paradigma iguala os não iguais no acesso às carreiras científicas e tecnológicas. Urge mudar esse comportamento $e$ apresentar os dados do desempenho científico e tecnológico na perspectiva de gênero para que os novos papéis possam emergir na sociedade.

A análise mostrou uma transformação nesses oito anos que este estudo investigou: a revolução feminista que empurrou as mulheres para as universidades e para a vida profissional aparece nos dados da biblioteca SciELO, as mulheres representam 32,28\% dos autores identificados. A ciência avança e a participação feminina também e no futuro próximo haverá cientistas de ambos os sexos e a ciência perderá a imagem misógina atualmente dominante. Mas, é preciso lembrar que as mulheres ainda continuam sujeitas a padrões diferenciados por gênero na escolha de carreiras profissionais próximas do estereótipo do ser mulher. As mudanças lentamente vão legitimando-se e por isso é necessário que estudos e pesquisas desmistifiquem a imagem partida feminina e que a ciência não é um lugar exclusivo dos homens, mas de quem tem talento para desempenhar estas funções, seja homem ou mulher.

\section{Referências bibliográficas}

BRASIL. Ministério de Ciência e Tecnologia. Livro Verde, 2001 e Livro Branco, 2002, Indicadores Nacionais de Ciência \& Tecnologia 2002, 2004.

Conselho Nacional de Desenvolvimento Científico e Tecnológico (CNPq), Plataforma LATTES. 
BRUSCHINI, C. \& LOMBARDI, M.R. Médicas, arquitetas, advogadas e engenheiras: mulheres em carreiras profissionais de prestígio. Revista de Estudos Feministas, vol. 7, n's 1/2, IFCS/UFRJ e CFCH/UFSC, 1999.

CARNEIRO Jr., Sandoval \& LOURENÇO, Ricardo. Pós-Graduação e Pesquisa na Universidade. In: VIOTTI, E.B. \& MACEDO, M. M. (orgs.) Indicadores de Ciência, Tecnologia e Inovação no Brasil. Campinas, Editora da UNICAMP, 2003.

COLES, J. e EALES, N.B. The history of comparative anatomy: a statistical analysis of scientific literature. Science Progress, vol. 11, 1917, pp.578-96.

COSTA, Ana Alice A. e SARDENBERG, Cecilia M.B. (orgs.) Feminismo, Ciência e Tecnologia, Salvador. Rede Feminista Norte e Nordeste de Estudos e Pesquisa sobre Mulher e Relações de Gênero (REDOR), Núcleo de Estudos Interdisciplinares sobre a Mulher (NEIM), Universidade Federal da Bahia, vol. 8, Coleção Bahianas, 2002.

FAPESP, Fundação de Amparo à Pesquisa do Estado de São Paulo. Scientific Electronic Library Online (SciELO). Site acessado ao longo dos meses de outubro/dezembro de 2005 e de janeiro a abril de 2006.

HARDING, Sandra. Is science multi-cultural? Postcolonialisms, feminisms, and epistemologies. Bloomington e Indianápolis, Indiana University Press, 1998.

LANDI, Francisco Romeu. Indicadores de Ciência, Tecnologia e Inovação em São Paulo - 2001. São Paulo, FAPESP, Fundação de Amparo à Pesquisa do Estado de São Paulo, 2002.

LETA, Jacqueline. As mulheres na ciência brasileira: crescimento, contrastes e um perfil de sucesso. Estudos Avançados 17 (49), São Paulo, 2003.

LETA, J. \& CRUZ, Carlos Henrique de Brito. A produção científica Brasileira. In: VIOTTI, E.B. \& MACEDO, M. M. (orgs.) Indicadores de Ciência, Tecnologia e Inovação no Brasil. Campinas, Editora da UNICAMP, 2003.

LOMBARDI, M.R. Mulheres engenheiras no mercado de trabalho brasileiro: qual seu lugar? In: GALEAZZI, Irene M.S. (ed.) Mulher e 
A produção científica brasileira no feminino

Trabalho. FEE, FGTAS/SINE-RS, DIEESE SEADE-SP, FAT/MTE PMPA, 2004.

LOPES, Maria Margaret. "Aventureiras" nas ciências: refletindo sobre gênero e história das ciências naturais no Brasil. Cadernos Pagu (10), Núcleo de Estudos de Gênero - Pagu/ Unicamp, 1998.

. A Ciência não é uma jovem de costumes fáceis: aspectos das relações de gênero na História da Ciência no Brasil. Cadernos IG/UNICAMP, vol.2, n 1, 1992.

MELO, Hildete Pereira de e LASTRES, H.M.M. Ciência e tecnologia numa perspectiva de gênero: o caso do CNPq. In: SANTOS, Lucy W. dos. (org.) Ciência, Tecnologia e Gênero: desvelando o feminino na construção do conhecimento. Londrina, Instituto Agronômico do Paraná (IAPAR), 2006.

MELO, Hildete Pereira de; LASTRES, Helena M.M.; MARQUES, Teresa C. de N. Gênero no sistema de ciência, tecnologia e inovação no Brasil. Revista Gênero, NUTEG/UFF, vol. 4, n 2, $1^{\circ}$ semestre de 2004.

MELO, Hildete Pereira de \& CASEMIRO, M.C.P. A Ciência no Feminino: uma análise da Academia Nacional de Medicina e da Academia Brasileira de Ciência. Revista Rio de Janeiro, UERJ/Fiocruz, $\mathrm{n}^{\circ}$ 11, set-dez 2003.

PEREZ SEDENO, Eulália. (ed.) Las mujeres em el sistema de Ciência y Tecnologia - Estudios de casos. Cuadernos de Iberoamérica, Organización de Estados Iberoamericanos (OEI), 2001.

Revista Ciência da Informação. vol. 27, n 2, 1998. Site www.scielo.br, acessado no dia 11 de maio de 2006.

RODRIGUEZ-SALA, M.L. e ZUBIETA GARCIA, Judith. (coord.) Mujeres em la Ciência y la Tecnologia: Hispanoamérica y Europa. México, Universidad Nacional Autônoma de México, Memórias 2, 2005.

SARDENBERG, Cecilia M.B. Da crítica feminista à Ciência a uma Ciência Feminista?. In: COSTA, A.A. e SARDENBERG, C.M.B. (orgs.) Feminismo, Ciência e Tecnologia. Salvador, Rede Feminista Norte e Nordeste de Estudos e Pesquisa sobre Mulher e Relações de Gênero (REDOR), Núcleo de Estudos Interdisciplinares sobre a Mulher (NEIM), Universidade Federal da Bahia, vol.8 Coleção Bahianas, 2002.

SCHIEBINGER, Londa. O Feminismo mudou a Ciência? Bauru-SP, EDUSC, 2001. 
Hildete Melo e André Oliveira

TABAK, F. O laboratório de Pandora - Estudos sobre ciência no feminino. Rio de Janeiro, Garamond Universitária, 2002.

TOSI, L. Mulher e Ciência: a revolução científica, a caça às bruxas e a ciência moderna. Cadernos Pagu (10), Núcleo de Estudos de Gênero - Pagu/Unicamp, 1998.

VELHO, Lea e LEÓN, E. A construção social da produção científica por mulheres. Cadernos Pagu (10), Núcleo de Estudos de Gênero Pagu/Unicamp, 1998.

VIOTTI, E.B. Fundamentos e Evolução dos Indicadores de CT\&I. In: VIOTTI, E.B. \& MACEDO, M. de M. (orgs.) Indicadores de Ciência, Tecnologia e Inovação no Brasil. Campinas, Editora da UNICAMP, 2003. 